\title{
Reconstrucción de la pared abdominal en pacientes con inmunosupresión: experiencia en una institución de alta complejidad
}

\author{
Reconstruction of the abdominal wall in patients with immunosuppression: \\ experience in a high complexity institution
}

\author{
Juan Manuel Márquez-Duque $\mathbb{D}^{\mathbb{D}}$, Paulo Andrés Cabrera-Rivera² $\mathbb{D}$
}

1 Médico, residente de Cirugía general, Universidad de la Sabana, Chía, Colombia.

2 Médico, especialista en Cirugía general, Fundación Cardioinfantil-Instituto de Cardiología, Bogotá D.C., Colombia.

\section{Resumen}

Introducción. Los pacientes con inmunosupresión llevados a cirugía abdominal convencional tienen un mayor riesgo de desarrollar hernias incisionales en el posoperatorio, y cuando ellas ocurren, es necesario individualizar el procedimiento quirúrgico de elección, según las características anatómicas, fisiológicas y clínicas de cada paciente. Este estudio describe las características demográficas y clínicas de los pacientes con inmunosupresión, llevados a reconstrucción de la pared abdominal, y sus desenlaces después de 30 días del procedimiento quirúrgico.

Métodos. Serie de casos de pacientes con inmunosupresión llevados a reconstrucción de la pared abdominal, en un centro especializado de cuarto nivel de complejidad. Se incluyeron pacientes mayores de 18 años, operados en el período de enero de 2016 a diciembre de 2019.

Resultados. Se presenta una serie de 18 pacientes, cinco $(27,7 \%)$ con algún tipo de inmunosupresión primaria y 13 (72,2 \%) con algún tipo de inmunosupresión secundaria. La edad promedio fue de 56 años, 11 (61 \%) fueron mujeres, el peso promedio de los participantes fue de 73,3 kg. Se encontraron complicaciones en ocho pacientes $(44,4 \%)$. Dos pacientes requirieron manejo en la Unidad de Cuidados Intensivos, por un máximo de tres días. Ninguno de los pacientes presentó recidiva de la hernia ni mortalidad.

Discusión. La reconstrucción de la pared abdominal en pacientes inmunosuprimidos representa un reto para cualquier equipo quirúrgico debido a las condiciones especiales de los pacientes y a las variables asociadas al procedimiento. Las tasas de recidiva y de complicaciones de este estudio, se asemejan a las descritas en la literatura.

Palabras clave: pared abdominal; inmunosupresión; reconstrucción; técnica; cirugía.

Fecha de recibido: 10/09/2020 - Fecha de aceptación: 26/03/2021 - Fecha de publicación en línea: 22/07/2021

Correspondencia: Juan Manuel Márquez-Duque, Universidad de la Sabana, Campus del Puente del Común, Km. 7, Autopista Norte de Bogotá. Chía, Colombia. Teléfono: +57(1) 8615555. Correo electrónico: juanmanuel-marquez@hotmail.com

Citar como: Márquez-Duque JM, Cabrera-Rivera PA. Reconstrucción de la pared abdominal en pacientes con inmunosupresión: experiencia en una institución de alta complejidad. Rev Colomb Cir. 2021;36:657-65. https://doi.org/10.30944/20117582.740

Este es un artículo de acceso abierto bajo una Licencia Creative Commons - BY-NC-ND https://creativecommons.org/licenses/by-ncnd/4.0/deed.es 


\begin{abstract}
Introduction. Immunosuppressed patients undergoing conventional abdominal surgery have a higher risk of developing incisional hernias postoperatively, and when they do occur, it is necessary to individualize the surgical procedure of choice, according to the anatomical, physiological and clinical characteristics of each patient. This study describes the demographic and clinical characteristics of immunosuppressed patients, who underwent abdominal wall reconstruction and their outcomes 30 days after the surgical procedure.
\end{abstract}

Methods. Series of cases of patients with immunosuppression underwent abdominal wall reconstruction in a specialized center of fourth level of complexity. Patients older than 18 years, operated on from January 2016 to December 2019, were included.

Results. A series of 18 patients is analyzed, five (27.7\%) with some type of primary immunosuppression and 13 (72.2\%) with some type of secondary immunosuppression. The average age was 56 years, 11 (61\%) were women, the average weight of the participants was $73.3 \mathrm{~kg}$. Complications were found in eight patients (44.4\%). Two patients required management in the ICU, for a maximum of three days. None of the patients had hernia recurrence or mortality.

Discussion. Reconstruction of the abdominal wall in immunosuppressed patients represents a challenge for any surgical team due to the special conditions of the patients and the variables associated with the procedure. The recurrence and complication rates in this study are similar to those described in the literature.

Keywords: abdominal wall; immunosuppression; reconstruction; technique; surgery.

\section{Introducción}

Las hernias incisionales (HI) se definen como "defectos en la pared abdominal alrededor de cicatrices posoperatorias, perceptibles o palpables mediante el examen clínico o mediante estudios de imágenes" ${ }^{1}$. Las HI son una causa importante de morbilidad, comprometen la calidad de vida de los pacientes y tienen un impacto económico que alcanza los tres billones de dólares en sólo sobrecostos en Estados Unidos de América ${ }^{2}$, donde, según el Center for Disease Control, se realizan cinco millones de laparotomías anualmente, con una tasa de incidencia de HI que se encuentra entre el $2 \%$ y el $20 \%$, y se realizan aproximadamente 350.000 herniorrafias al año, de las cuales, al menos, 200.000 están relacionadas con incisiones previas ${ }^{3}$.

La predisposición para la formación de una HI, está dada por factores de riesgo sistémicos y locales, ampliamente evaluados en estudios prospectivos y retrospectivos. Dentro de las predisposiciones sistémicas se encuentran el estado nutricional, la ictericia, la diabetes mellitus, la obesidad, el tabaquismo, alteración de las proteínas séricas, edad, género, uso crónico de esteroides y estados de inmunosupresión del paciente ${ }^{1-7}$. Esta última cobra relevancia, al considerar que estudios previos han mostrado que entre un $2 \%$ y un $10 \%$ de todos los pacientes llevados a una Reparación de Hernia Ventral (RHV), padecen de alguna clase de inmunosupresión (primaria o secundaria) al momento de la intervención quirúrgica. Se ha encontrado que, en pacientes trasplantados (estado común relacionado con la inmunosupresión secundaria), la prevalencia de $\mathrm{HI}$ alcanza hasta el $45 \%$ en algunas series $4,8,9$.

Las inmunodeficiencias son un grupo de enfermedades causadas por cambios cuantitativos o funcionales en los mecanismos a cargo de la respuesta inmune innata y adaptativa. Se estima que afectan a seis millones de personas a nivel mundial. Estos trastornos pueden ser de origen primario o secundario. Las inmunodeficiencias primarias son desórdenes inmunitarios heredados, derivados de mutaciones genéticas, que generalmente se diagnostican al nacer o en la infancia. Las inmunodeficiencias secundarias son el resultado de enfermedades o factores ambientales que debilitan el sistema inmune ${ }^{10}$. 
La inmunosupresión es considerada como un factor de riesgo para la aparición de HI, dado que altera la angiogénesis, la formación de colágeno, compromete las funciones proinflamatorias de las células que se encuentran en las heridas y, a su vez, disminuye la quimio-atracción de plaquetas, neutrófilos y macrófagos, que se relacionan con el proceso de cicatrización normal ${ }^{10}$. Los estados de inmunosupresión tienen una fisiología cicatricial alterada, que hace que los eventos adversos relacionados con la herida quirúrgica aumenten, causando a su vez un incremento en la morbilidad y la mortalidad, comprometiendo la calidad de vida de los pacientes, promoviendo estados de depresión, estrés postraumático, aumento de las incapacidades laborales y limitaciones funcionales ${ }^{10,11}$.

Los pacientes inmunosuprimidos con hernias incisionales se pueden dividir en dos grupos: pacientes con hernias simples, en quienes el espectro del tratamiento abarca desde cierres primarios hasta reparaciones con malla vía abierta y laparoscópica, según las características y el criterio del cirujano ${ }^{12}$, y pacientes con HI complejas o pérdida del domicilio, que requieren previamente el uso de neumoperitoneo preoperatorio progresivo o aplicación de toxina botulínica y, posteriormente, procedimientos quirúrgicos complejos, como la separación de componentes, la técnica de Rives-Stoppa, IPOM (Intraperitoneal Onlay Mesh) o TAR (Transversus Abdominis Release) ${ }^{8,13,14}$.

La variedad de técnicas disponibles para dar manejo a este tipo de pacientes es aún más amplia y se podría llegar a la conclusión de que, esta variabilidad es causa de la falta de consenso acerca de cuál es el manejo óptimo de los pacientes inmunosuprimidos, que requieren reconstrucciones complejas de la pared abdominal, y los desenlaces asociados a cada tipo de intervención ${ }^{13,15-19}$. A pesar de la falta de consenso sobre el tipo de reconstrucción, Daes ${ }^{20}$ plantea seis reglas básicas para la reconstrucción de la pared abdominal: 1) Cierre primario de los defectos fasciales bajo tensión fisiológica, 2) Amplio refuerzo protésico del saco visceral, 3) Fijación limitada del material protésico, 4) Colocación de mallas idealmente "sublay", 5) Abordaje mínimamente invasivo cuando sea posible y 6) Aplicación del protocolo ERAS (Enhanced Recovery After Surgery).

El objetivo de este estudio es publicar en Latinoamérica una de las primeras series de casos que describa las características demográficas y clínicas de los pacientes con antecedente de inmunosupresión, llevados a reconstrucción de la pared abdominal y sus desenlaces a 30 días luego de la cirugía.

\section{Métodos}

Estudio observacional de tipo serie de casos. Se recolectó de forma prospectiva una muestra de 21 pacientes con algún tipo de inmunosupresión documentada en la historia clínica, quienes fueron llevados a reconstrucción de la pared abdominal en la Fundación Cardioinfantil - Instituto de Cardiología, Bogotá, D.C., Colombia, entre enero de 2016 y diciembre de 2019. Los criterios de inclusión fueron:

1. Tener más de 18 años

2. Tener algún tipo de inmunosupresión al momento del procedimiento.

3. Haber sido llevados a reconstrucción de pared abdominal en la institución.

4. Tener un seguimiento postoperatorio institucional a 30 días.

5. Disponibilidad de la información de cada paciente en la base de datos.

De los 21 pacientes, 18 cumplieron con los criterios de selección.

La información recolectada permitió evaluar variables demográficas y perioperatorias como edad, sexo, tipo de inmunosupresión, localización de la hernia, tipo de malla utilizada, tiempo quirúrgico, requerimiento de Unidad de Cuidados Intensivos (UCI) posoperatoria y complicaciones (infección del sitio operatorio [ISO], recidiva, mortalidad) derivadas del procedimiento y ocurridas en los primeros 30 días después de llevada a cabo la cirugía. Todas las complicaciones fueron verifi- 
cadas mediante la revisión de las historias clínicas. Para la categorización de las HI, se utilizó la clasificación de la Sociedad Europea de la Hernia, que determina las nominaciones de M1 a M5 cuando son sobre la línea media y de L1 a L4 cuando son laterales ${ }^{21}$.

\section{Procedimiento quirúrgico}

Antes de la cirugía todos los pacientes fueron evaluados por un equipo multidisciplinario, para determinar el estado médico y nutricional del paciente y asegurar la viabilidad del procedimiento quirúrgico. Los pacientes fueron sometidos a alguno de los siguientes procedimientos con el fin reconstruir la pared abdominal:

1. IPOM, técnica laparoscópica: en posición decúbito supino, inserción de trócares bajo técnica abierta, insuflación de la cavidad abdominal con $\mathrm{CO}_{2}$, corrección del defecto herniario y fijación de la malla de forma intraperitoneal.

2. Cierre primario, con o sin uso de malla, técnica abierta: disección de los colgajos "dermograsos", afrontamiento delínea media y colocación de la malla sobre la fascia ("onlay"), en los pacientes que así lo requerían.

3. SAC (separación anterior de componentes) / Ramírez-Carbonell, técnica abierta: Fasciotomía longitudinal oblicua externa, liberación del músculo oblicuo externo, disección del plano avascular entre músculos y avance medial con afrontamiento de línea media y colocación de malla en este plano. Disección subcutánea extensa, con exposición de la línea semilunar, afrontamiento de los bordes del oblicuo mayor a la malla, cubriendo los bordes de la malla y permitiendo al oblicuo mayor continuar como una unidad funcional.

4. Rives-Stoppa, técnica abierta: Liberación del músculo recto en su vaina posterior, afrontamiento en la línea media de la vaina muscular posterior. Colocación de malla en el espacio retro-rectal, el cual es vascularizado, favoreciendo la adecuada incorporación de la malla.
5. TAR (Transversus Abdominis Release), técnica abierta: Disección de la línea arcuata de abajo hacia arriba, disección del espacio preperitoneal desde el espacio de Bogros, disección y liberación del músculo transverso del abdomen, afrontamiento de la línea media, colocación de la malla cubriendo la línea media, músculos psoas, espacio retroperitoneal lateralmente, espacios de Retzius y Bogros.

Durante el postoperatorio todos los pacientes fueron valorados por un equipo multidisciplinario compuesto por los grupos de clínica de dolor, pared abdominal, trasplantes y reumatología, terapia respiratoria y física y soporte nutricional. El seguimiento ambulatorio fue realizado por el mismo grupo.

\section{Análisis estadístico}

Para el análisis descriptivo se utilizó el software RStudio (R versión 3.0.1; RStudio, Boston, MA). Las variables continuas se resumieron por mediana (rango intercuartílico [RIC] percentil 25, percentil 75) y las variables categóricas se informaron como n (\%) en tablas de frecuencia.

\section{Resultados}

El promedio de edad fue de 56 años (DE: 13,4) y los participantes tuvieron edades entre 18 y 74 años (tabla 1). El índice de masa corporal (IMC) promedio fue de $20,9 \mathrm{~kg} / \mathrm{m}^{2}$, con un mínimo de 18,1 y un máximo de $42,6 \mathrm{~kg} / \mathrm{m}^{2}$. La cirugía mayor correspondió al antecedente más frecuente y la hipertensión arterial fue la comorbilidad más usual. La ubicación más frecuente de la HI fue la línea media, con un 55,5\% $(n=10)$, seguido de la incisión de Chevron, en un 22,2 \% ( $n=4)$.

En cuanto al tipo de reconstrucción de pared abdominal (tabla 2), la separación anterior de componentes tipo Carbonell y la eventrorrafia por laparoscopia (IPOM) fueron los procedimientos más frecuentes, cada uno en cinco pacientes $(27,7 \%)$. El tiempo quirúrgico promedio de los procedimientos fue de 146,9 minutos (RIC: 86,7 ), siendo 44 minutos el tiempo mínimo y 245 minutos el tiempo máximo de cirugía. 
El tiempo promedio de estancia hospitalario fue de 10,7 días, siendo 34 días la estancia hospitalaria máxima y 3 días la estancia hospitalaria mínima. De los 18 pacientes, 2 (11,1 \%) requirieron manejo postoperatorio en UCI. Uno de los pacientes que requirió UCI tuvo una estancia de tres días, con necesidad de soporte vasopresor y ventilatorio por un día. El otro paciente sólo requirió vigilancia postoperatoria en UCI por un día.

En la tabla 3 se muestran los desenlaces clínicos de los pacientes. Se presentaron complicaciones de algún tipo en ocho pacientes $(44,4 \%)$, las cuales fueron categorizadas según la clasificación de Clavien-Dindo ${ }^{22}$. Todos los pacientes con ISO

Tabla 1. Características sociodemográficas de los pacientes con inmunosupresión llevados a reconstrucción de la pared abdominal en la Fundación Cardioinfantil - Instituto de Cardiología, Bogotá, D.C., Colombia. 2016-2019.

\begin{tabular}{|c|c|}
\hline Características & $\begin{array}{c}\text { Serie de casos } \\
(n=18)\end{array}$ \\
\hline Edad (años), media \pm DE & $56 \pm 13,4$ \\
\hline \multicolumn{2}{|l|}{ Sexo (\%) } \\
\hline Mujer & $11(61,1 \%)$ \\
\hline Hombre & $7(38,8 \%)$ \\
\hline Peso, media $\pm \mathrm{DE}(\mathbf{k g})$ & $73,3 \pm 19,1$ \\
\hline \multicolumn{2}{|l|}{ Tipo de inmunosupresión } \\
\hline Primaria & $5(27,7 \%)$ \\
\hline Secundaria & $13(72,2 \%)$ \\
\hline \multicolumn{2}{|l|}{ Causa de inmunosupresión } \\
\hline Trasplante hepático & $4(22,2 \%)$ \\
\hline Quimio/radioterapia & $3(16,7 \%)$ \\
\hline Trasplante renal & $2(11,1 \%)$ \\
\hline Artritis reumatoide & $1(5,6 \%)$ \\
\hline Cirrosis hepática de causa no clara & $1(5,6 \%)$ \\
\hline Colitis ulcerativa & $1(5,6 \%)$ \\
\hline Falla hepática & $1(5,6 \%)$ \\
\hline Hepatitis autoinmune & $1(5,6 \%)$ \\
\hline Insuficiencia renal crónica & $1(5,6 \%)$ \\
\hline Lupus eritematoso sistémico & $1(5,6 \%)$ \\
\hline Trasplante cardiaco & $1(5,6 \%)$ \\
\hline Trasplante pulmonar & $1(5,6 \%)$ \\
\hline \multicolumn{2}{|c|}{ Consumo de medicamentos inmunosupresores } \\
\hline $\mathrm{Si}$ & $12(66,6 \%)$ \\
\hline No & $6(33,3 \%)$ \\
\hline
\end{tabular}

fueron tratados de forma exitosa con el manejo convencional. Posterior al egreso hospitalario, siete pacientes $(38,9 \%)$ reingresaron durante los siguientes 30 días a la institución. De ellos, cuatro por causas asociadas al procedimiento quirúrgico. En los otros tres pacientes el reingreso no se relacionó con el antecedente quirúrgico. No ocurrió ninguna muerte.

\section{Discusión}

El manejo de las HI complejas en pacientes inmunosuprimidos, representa un reto para los grupos que realizan el tratamiento quirúrgico de estos procedimientos, calificados como de alta complejidad por su dificultad técnica y por las características de los pacientes, factores que se asocian con desenlaces negativos según algunos reportes ${ }^{4,9,23}$. La falta de consenso en el tipo de procedimiento quirúrgico de elección para estos

Tabla 2. Características prequirúrgicas y quirúrgicas de los pacientes con inmunosupresión llevados a reconstrucción de la pared abdominal en la Fundación Cardioinfantil - Instituto de Cardiología, Bogotá, D.C., Colombia. 2016-2019.

\begin{tabular}{lc}
\hline \multicolumn{1}{c}{ Características } & $\begin{array}{c}\text { Serie de casos } \\
(\mathbf{n = 1 8 )}\end{array}$ \\
\hline ASA (American Society of & \\
Anesthesiologists) & \\
ASA II & $6(33,3 \%)$ \\
ASA II & $12(66,6 \%)$ \\
Tipo de reconstrucción pared & \\
abdominal N ${ }^{1}$ & \\
SAC Carbonell & $5(27,7 \%)$ \\
IPOM & $5(27,7 \%)$ \\
Rives Stoppa & $4(22,2 \%)$ \\
Cierre primario & $6(16,6 \%)$ \\
TAR & $1(5,6 \%)$ \\
Tipo de reconstrucción pared & \\
abdominal N ${ }^{2}$ & \\
SAC Carbonell & $1(5,6 \%)$ \\
Tipo de malla empleada & \\
Polipropileno & $11(61,1 \%)$ \\
Symbotex ${ }^{\text {TM }}$ & $5(27,7 \%)$ \\
No uso de malla & $2(11,1 \%)$ \\
Tiempo quirúrgico & 146,9 min \\
\hline DE: desviación estándar; SAC: separación anterior de componentes; \\
IPOM: intraperitoneal Onlay Mesh; TAR: Transversus Abdominis \\
Release
\end{tabular}


casos demuestra la necesidad de continuar investigando este tópico e informando los desenlaces clínicos relevantes ${ }^{13}$.

Las características sociodemográficas de los estudios reportados en series similares son variables. Al comparar este estudio con los resultados reportados en la literatura, se encuentran similitudes como la edad media, que se ubicó en 56,1 años en esta serie y en 54,9 para los resultados de Lafitic ${ }^{24} \mathrm{y}$ otros estudios que reportaron

Tabla 3. Desenlaces clínicos de los pacientes del estudio.

\begin{tabular}{|c|c|}
\hline Características & $\begin{array}{l}\text { Serie de casos } \\
\qquad(n=18)\end{array}$ \\
\hline $\begin{array}{l}\text { Estancia hospitalaria promedio (días) } \\
\text { Estancia hospitalaria en UCl }\end{array}$ & 10,7 días +/- 7 \\
\hline $\mathrm{Si}$ & $2(11,1 \%)$ \\
\hline No & $16(89,9 \%)$ \\
\hline Estancia promedio en UCl (días) & 2 días \\
\hline \multicolumn{2}{|l|}{ Requerimiento de soporte ventilatorio } \\
\hline $\mathrm{Si}$ & $1(5,6 \%)$ \\
\hline $\begin{array}{l}\text { Tiempo de requerimiento soporte } \\
\text { ventilatorio (días) }\end{array}$ & 1 día \\
\hline \multicolumn{2}{|l|}{ Requerimiento de soporte vasopresor } \\
\hline $\mathrm{Si}$ & $1(5,6 \%)$ \\
\hline $\begin{array}{l}\text { Tiempo de requerimiento soporte } \\
\text { ventilatorio (días) }\end{array}$ & 1 día \\
\hline \multicolumn{2}{|l|}{ Clasificación Clavien Dindo } \\
\hline Grado I & $2(11,1 \%)$ \\
\hline Grado II & 0 \\
\hline Grado III & $6(33,3 \%)$ \\
\hline Grado IIla & $3(16,7 \%)$ \\
\hline Grado IIIb & $3(16,7 \%)$ \\
\hline Grado IV & 0 \\
\hline Grado V & 0 \\
\hline \multicolumn{2}{|l|}{ Infección sitio operatorio (ISO) } \\
\hline $\mathrm{Si}$ & $4(22,2 \%)$ \\
\hline No & $14(77,8 \%)$ \\
\hline \multicolumn{2}{|l|}{ Tipo de ISO } \\
\hline Incisional superficial & $2(11,1 \%)$ \\
\hline Órgano espacio & $2(11,1 \%)$ \\
\hline \multicolumn{2}{|l|}{ Reingreso a los 30 días } \\
\hline $\mathrm{Si}$ & $4(22,2 \%)$ \\
\hline \multicolumn{2}{|l|}{ Recidiva de la hernia } \\
\hline No & $18(100 \%)$ \\
\hline \multicolumn{2}{|l|}{ Mortalidad derivada de la cirugía } \\
\hline No & $18(100 \%)$ \\
\hline
\end{tabular}

medias de 55,5 y 47,1 años 9,23. En el caso de las comorbilidades de los pacientes llevados al procedimiento, la literatura describe la prevalencia de Diabetes Mellitus entre 20-30\%, la cual es similar a la de este estudio $(22,2 \%)^{9,23,24}$. La comparación de otras comorbilidades, como el antecedente de EPOC y cáncer, que en este estudio fueron de 5,6 \% y 27,8 \% respectivamente, tuvo un comportamiento similar a las prevalencias reportadas por la literatura. El IMC fue otro de los factores en los que se encontró similitud entre esta serie y las otras reportadas, con valores entre 25 y $33 \mathrm{~kg} / \mathrm{m}^{2}$ 9,23,24.

Sin embargo, para algunas características como el sexo y el antecedente de enfermedades cardiovasculares de los pacientes operados, se encontraron diferencias con respecto a la literatura. En el caso del sexo, esta serie presentó una mayor proporción de mujeres $(61,1 \%)$ en comparación con otras series. En el caso del antecedente de enfermedades cardiovasculares, se encontró una prevalencia del $50 \%$ en esta serie, la cual es mayor en comparación con el porcentaje reportado por Latifi $^{24}$. Lo anterior se puede deber a que nuestra institución es un centro de referencia para el manejo de patologías cardiovasculares.

En esta serie de 18 casos de pacientes inmunosuprimidos y llevados a reconstrucción de pared, no hubo ninguna recidiva dentro de los primeros 30 días y hubo una tasa general de complicaciones del 44,4\%. Estos valores son similares a los reportados por Zolper ${ }^{13}$, con una tasa de recidivas de 5,4 \% y general de complicaciones del 31,4\%. Similares también a lo informado por Latifi ${ }^{24}$, en pacientes con reconstrucciones de pared abdominal complejas, realizadas en la población general, con valores de morbilidad del 35,8 \%. Para el caso de las recidivas, en las que los valores reportados en la población general se encuentran entre el $6 \%$ y el $29 \%$, se puede observar que el desenlace planteado en esta serie de pacientes inmunosuprimidos es mejor que lo reportado por otros autores ${ }^{25,26}$.

En la evolución postoperatoria, el número de complicaciones asociadas no es el único factor relevante, la gravedad de estas también es impor- 
tante. La serie de 30 pacientes reportada por Scholer ${ }^{27}$, en la que se evaluaron las complicaciones según la clasificación de Clavien-Dindo, mostró una tasa de complicaciones grado III del $23,3 \%$ y grado IV del $30 \%$. En este estudio las complicaciones grado III fueron del $33 \%$ y no hubo grado IV. Nuestros resultados muestran un mejor desenlace del procedimiento quirúrgico, a pesar de la condición de inmunosupresión de nuestra población.

El uso de malla en la reconstrucción de la pared abdominal se ha asociado a la disminución de las recidivas de las $\mathrm{HI}^{2,28}$. La siguiente discusión, por ende, sería el tipo de malla a utilizar. Algunos autores han reportado la ventaja, en pacientes inmunosuprimidos, del uso de mallas sintéticas sobre las mallas biológicas debido a que muestran una menor tasa de complicaciones ${ }^{13,29,30}$. Los resultados encontrados en este estudio muestran una situación similar, en el $20 \%$ de los pacientes en los que se utilizó malla sintética ocurrieron complicaciones, en comparación con un 54,4\% de complicaciones cuando se utilizó una malla biológica. Por otro lado, el uso de mallas biológicas, según lo reportado por otros autores, puede aumentar la tasa de recidivas, pero en esta serie no hubo recurrencia en ninguno de los pacientes ${ }^{14,28}$.

Una de las limitaciones de este estudio es el tamaño de la muestra, que puede representar dificultades para generalizar los resultados obtenidos o establecer relaciones causales. Para evitar fallos en el registro, se realizó la revisión de la historia clínica de cada uno de los pacientes de forma individual y en caso de dudas, se verificó con el paciente la información por vía telefónica. Hasta donde conocemos, esta serie es la primera en Latinoamérica en la que se describen, tanto las variables sociodemográficas como los desenlaces clínicos, en pacientes inmunosuprimidos que han sido llevados a reconstrucción de la pared abdominal. Por esta razón, el registro de los desenlaces fue riguroso, logrando indagar a profundidad sobre los desenlaces relevantes según el tipo de procedimiento quirúrgico y el tipo de malla que se usó, para su correcta comparación.

\section{Conclusión}

La reconstrucción de la pared abdominal en pacientes inmunosuprimidos es una opción de manejo compleja dadas las condiciones especiales, tanto de los pacientes elegidos como de otras variables asociadas de forma inherente al procedimiento. Esto representa un reto para cualquier equipo quirúrgico y por estas razones es probable que no todas las instituciones se encuentren preparadas para dar un manejo integral y óptimo a este tipo de pacientes. Existe la necesidad de continuar haciendo investigación en este campo específico para recopilar más información y ofrecer las mejores opciones a pacientes que presenten esta patología.

\section{Cumplimiento de normas éticas}

Consentimiento informado: De acuerdo con las normas de investigación en seres humanos según lo dispuesto en el artículo 11 de la Resolución No. 8430 de 1993 del Ministerio de Salud de Colombia y en la declaración de Helsinki de 2000, esta es una investigación sin riesgo. Se tomaron los datos registrados en la historia clínica de la institución y mediante una llamada telefónica se realizó una breve encuesta. Todos los datos fueron organizados y analizados de forma anónima, en ningún momento se divulgaron datos que hayan permitido la identificación de los pacientes manteniendo a los participantes en el anonimato. El presente trabajo fue presentado y aprobado por el Comité de Ética y de Investigaciones de la institución

Conflictos de interés: Para el presente estudio ninguno de los autores tiene conflictos de interés.

Fuente de financiación: El presente estudio contó con la financiación de la Fundación Cardioinfantil - Instituto de Cardiología, Bogotá, D.C., Colombia.

\section{Contribución de los autores}

Concepción y diseño del estudio: Juan Manuel MárquezDuque, Paulo Andrés Cabrera-Rivera.

Adquisición de datos: Juan Manuel Márquez-Duque, Paulo Andrés Cabrera-Rivera.

Análisis e interpretación de datos: Juan Manuel MárquezDuque, Paulo Andrés Cabrera-Rivera.

Redacción del manuscrito: Juan Manuel Márquez-Duque, Paulo Andrés Cabrera-Rivera.

Revisión crítica: Juan Manuel Márquez-Duque, Paulo Andrés Cabrera-Rivera. 


\section{Referencias}

1. Bosanquet DC, Ansell J, Abdelrahman T, Cornish J, Harries R, Stimpson A, et al. Systematic review and meta-regression of factors affecting midline Incisional hernia rates: Analysis of 14.618 Patients. PLoS One. 2015;10:1-18.

https://doi.org/10.1371/journal.pone.0138745

2. Borab ZM, Shakir S, Lanni MA, Tecce MG, MacDonald J, Hope WW, et al. Does prophylactic mesh placement in elective, midline laparotomy reduce the incidence of incisional hernia? A systematic review and meta-analysis. Surg (United States). 2017;161:1149-63. https://doi.org/10.1016/j.surg.2016.09.036

3. Smith CT, Katz MG, Foley D, Welch B, Leverson GE, Funk $\mathrm{LM}$, et al. Incidence and risk factors of incisional hernia formation following abdominal organ transplantation. Surg Endosc. 2015;29:398-404.

https://doi.org/10.1007/s00464-014-3682-8

4. Haskins IN, Krpata DM, Prabhu AS, Tastaldi L, Perez AJ, $\mathrm{Tu} \mathrm{C}$, et al. Immunosuppression is not a risk factor for 30-day wound events or additional 30-day morbidity or mortality after open ventral hernia repair: An analysis of the Americas Hernia Society Quality Collaborative. Surg (United States). 2018;164:594-600. https://doi.org/10.1016/j.surg.2018.05.023

5. Broggi E, Bruyère F, Gaudez F, Desgrandchamps F. Risk factors of severe incisional hernia after renal transplantation: a retrospective multicentric case-control study on 225 patients. World J Urol. 2017;35:1111-7. https://doi.org/10.1007/s00345-016-1971-7

6. Fikatas P, Schoening W, Lee JE, Chopra SS, Seehofer D, Guckelberger 0 , et al. Incidence, risk factors and management of incisional hernia in a high volume liver transplant center. Ann Transplant. 2013;18:223-30. https://doi.org/10.12659/AOT.883914

7. Jairam AP, Timmermans L, Eker HH, Pierik REGJM, van Klaveren D, Steyerberg EW, et al. Prevention of incisional hernia with prophylactic onlay and sublay mesh reinforcement versus primary suture only in midline laparotomies (PRIMA): 2-year follow-up of a multicentre, double-blind, randomised controlled trial. Lancet. 2017;390:567-76. https://doi.org/10.1016/S0140-6736(17)31332-6

8. Lambrecht JR, Skauby M, Trondsen E, Vaktskjold A, Øyen OM. Laparoscopic repair of incisional hernia in solid organ-transplanted patients: The method of choice? Transpl Int. 2014;27:712-20. https://doi.org/10.1111/tri.12327

9. Ozgor D, Dirican A, Ates M, Yilmaz M, Isik B, Yilmaz S. Incisional hernia in recipients of adult to adult living donor liver transplantation. World J Surg. 2014;38:2122-5. https://doi.org/10.1007/s00268-014-2528-9

10. Fernandez J. Overview of immunodeficiency disorders. cleveland clinic lerner college of medicine at case western reserve university. Disponible en: https://www. msdmanuals.com/home/immune-disorders/immu- nodeficiency-disorders/overview-of-immunodeficiency-disorders. Fecha de consulta: 3 de julio de 2020.

11. Jensen KK, Munim K, Kjaer M, Jorgensen LN. Abdominal wall reconstruction for incisional hernia optimizes truncal function and quality of life: A prospective controlled study. Ann Surg. 2017;265:1235-40. https://doi.org/10.1097/SLA.0000000000001827

12. Eisenberg D, Popescu WM, Duffy AJ, Bell RL. Laparoscopic treatment of subxiphoid incisional hernias in cardiac transplant patients. J Soc Laparoendosc Surg. 2008;12:262-6. Disponible en https://pubmed.ncbi.nlm.nih.gov/18765049/

13. Zolper EG, Black CK, Wang CDJ, Kroemer MMAH. Long term outcomes of abdominal wall reconstruction using open component separation and biologic mesh in the liver, kidney, and small bowel transplant population. Hernia. 2020;:469-79. https://doi.org/10.1007/s10029-019-02117-1

14. Black CK, Zolper EG, Walters ET, Wang J, Martinez J, Tran A, et al. Utility of a modified components separation for abdominal wall reconstruction in the liver and kidney transplant population. Arch Plast Surg. 2019;46:462-9. https://doi.org/10.5999/aps.2018.01361

15. Chang EI, Galvez MG, Padilla BE, Freise CE, Foster RD, Hoffman WY. Ten-year retrospective analysis of incisional herniorrhaphy following renal transplantation. Arch Surg. 2011;146:21-5. https://doi.org/10.1001/archsurg.2010.305

16. Ayvazoglu Soy EH, Kirnap M, Yildırim S, Moray G, Haberal M. Incisional hernia after liver transplant. Exp Clin Transplant. 2017;15:185-9. https://doi:10.6002/ect.mesot2016.P65.

17. GerlachUA, Pascher A. Technical advances for abdominal wall closure after intestinal and multivisceral transplantation. Curr Opin Organ Transplant. 2012;17:258-67. https://doi.org/10.1097/MOT.0b013e3283534d7b

18. Carlsen BT, Farmer DG, Busuttil RW, Miller TA, Rudkin GH. Incidence and management of abdominal wall defects after intestinal and multivisceral transplantation. Plast Reconstr Surg. 2007;119:1247-55. https://doi.org/10.1097/01.prs.0000254401.33682.e9

19. Ooms LS, Verhelst J, Jeekel J, Ijzermans JN, Lange JF, Terkivatan T. Incidence, risk factors, and treatment of incisional hernia after kidney transplantation: An analysis of 1,564 consecutive patients. Surg (United States). 2016;159:1407-11. https://doi.org/10.1016/j.surg.2015.11.017

20. Daes J, Telem D. The Principled Approach to Ventral Hernia Repair. El abordaje basado en principios para el reparo de la hernia ventral. Rev Colomb Cir. 2019;34:25-8. https://doi.org/10.30944/20117582.94

21. De la Cuadra M, Jurado G, Moreno G, Romero M. Actualización Clasificación actual EHS. Cir Andaluza. 2013;24:225-227. Disponible en: https://www.asacirujanos.com/admin/upfiles/revista/2013/2013-vol24-n3-4-act2.pdf 
22. Clavien PA, Barkun J, De Oliveira ML, Vauthey JN, Dindo D, Schulick RD, et al. The clavien-dindo classification of surgical complications: Five-year experience. Ann Surg. 2009;250:187-96. https://doi.org/10.1097/SLA.0b013e3181b13ca2

23. Kuo SC, Lin CC, Elsarawy A, Lin YH, Wang SH, Wu YJ, et al. Laparoscopic repair of incisional hernia following liver transplantation-early experience of a single institution in Taiwan. Transplant Proc. 2017;49:1870-4. https://doi.org/10.1016/j.transproceed.2017.04.019

24. Latifi R, Samson DJ, Gogna S, Joseph BA. Perioperative complications of complex abdominal wall reconstruction with biologic mesh: A pooled retrospective cohort analysis of 220 patients from two academic centers. Int J Surg. 2020;74:94-9. https://doi.org/10.1016/j.ijsu.2019.12.035

25. MüllerV,LehnerM,Klein P,HohenbergerW,OttR.Incisional hernia repair after orthotopic liver transplantation: A technique employing an inlay/onlay polypropylene mesh. Langenbeck's Arch Surg. 2003;388:167-73. https://doi.org/10.1007/s00423-003-0384-4

26. Mekeel K, Mulligan D, Reddy KS, Moss A, Harold K. Laparoscopic incisional hernia repair after liver transplantation. Liver Transplant. 13;11:1576-81. https://doi.org/10.1002/lt.21290

27. Scholer AJ, Oliver JB, Rosado J, Patel JS, Lynch LJ, Spiegler KM, et al. Science direct abdominal wall reconstruction after cytoreduction chemotherapy. J Surg Res. 2017;221:266-74.

https://doi.org/10.1016/j.jss.2017.08.002
28. Scheuerlein H, Thiessen A, Schug-Pass C, Köckerling F. What do we know about component separation techniques for abdominal wall hernia repair? Front Surg. 2018;5:1-8. https://doi.org/10.3389/fsurg. 2018.00024

29. Brewer MB, Rada EM, Milburn ML, Goldberg NH, Singh DP, Cooper M, et al. Human acellular dermal matrix for ventral hernia repair reduces morbidity in transplant patients. Hernia. 2011;15:141-5. https://doi.org/10.1007/s10029-010-0748-y

30. Santangelo ML, Carlomagno N, Spiezia S, Danilo Palmieri D, Clemente M, Piantadosi MP, et al. Use of biological prostheses in transplant patients with incisional hernias. Preliminary experience. Ann Ital Chir. 2013;84:4715. Disponible en: http://www.ncbi.nlm.nih.gov/pubmed/23135483

31. Poulose BK, Shelton J, Phillips S, Moore D, Nealon W, Penson D, et al. Epidemiology and cost of ventral hernia repair: Making the case for hernia research. Hernia. 2012;16:179-83. https://doi.org/10.1007/s10029-011-0879-9

32. Van Ramshorst GH, Eker HH, Hop WCJ, Jeekel J, Lange JF. Impact of incisional hernia on health-related quality of life and body image: A prospective cohort study. Am J Surg. 2012;204:144-50. https://doi.org/10.1016/j.amjsurg.2012.01.012

33. Aliotta RE, Gatherwright J, Krpata D, Rosenblatt S, Rosen M, Gurunluoglu R. Complex|, harnessing the power of a specialized multidisciplinary team to improve pain and quality of life. Hernia. 2019;23:205-15. https://doi.org/10.1007/s10029-019-01916-w 